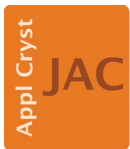

JOURNAL OF

APPLIED

CRYSTALLOGRAPHY

ISSN 1600-5767

Received 20 October 2015

Accepted 27 April 2016

Edited by E. P. Gilbert, ANSTO, Kirrawee DC, Australia

Keywords: polarized neutron reflectometry; applied magnetic fields; Zeeman corrections; non-collinear magnetization.

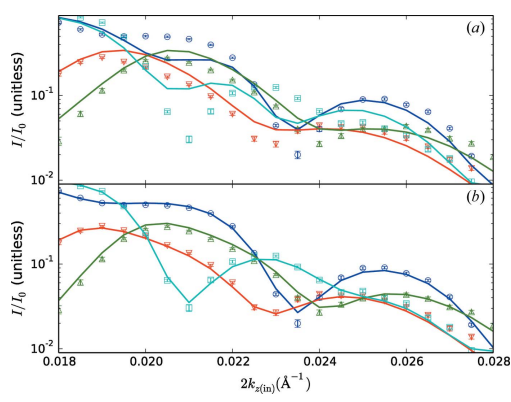

OPEN $\odot$ ACCESS

\section{Measurement and modeling of polarized specular neutron reflectivity in large magnetic fields}

\author{
Brian B. Maranville, ${ }^{\mathrm{a} *}$ Brian J. Kirby, ${ }^{\mathrm{a}}$ Alexander J. Grutter, ${ }^{\mathrm{a}}$ Paul A. Kienzle, \\ Charles F. Majkrzak, ${ }^{a}$ Yaohua Liu ${ }^{b}$ and Cindi L. Dennis ${ }^{c}$
}

\begin{abstract}
${ }^{\mathbf{a}}$ NIST Center for Neutron Research, 100 Bureau Drive, Gaithersburg, MD 20899, USA, ${ }^{\mathbf{b}}$ Quantum Condensed Matter Division, Oak Ridge National Laboratory, Oak Ridge, TN 37831, USA, and ${ }^{\mathbf{c}}$ NIST Material Measurement Laboratory, 100 Bureau Drive, Gaithersburg, MD 20899, USA. *Correspondence e-mail: brian.maranville@nist.gov
\end{abstract}

The presence of a large applied magnetic field removes the degeneracy of the vacuum energy states for spin-up and spin-down neutrons. For polarized neutron reflectometry, this must be included in the reference potential energy of the Schrödinger equation that is used to calculate the expected scattering from a magnetic layered structure. For samples with magnetization that is purely parallel or antiparallel to the applied field which defines the quantization axis, there is no mixing of the spin states (no spin-flip scattering) and so this additional potential is constant throughout the scattering region. When there is non-collinear magnetization in the sample, however, there will be significant scattering from one spin state into the other, and the reference potentials will differ between the incoming and outgoing wavefunctions, changing the angle and intensities of the scattering. The theory of the scattering and recommended experimental practices for this type of measurement are presented, as well as an example measurement.

\section{Introduction}

Polarized specular neutron reflectometry measurements require at least a small magnetic field to be applied throughout the measurement apparatus, in order to maintain a well defined neutron quantization axis. In addition, a larger field is often applied at the sample position in order to manipulate the magnetic state of the sample (Majkrzak et al., 2006). The difference in the Zeeman energy for a spin-up versus a spindown neutron can lead to observable shifts in both the angle and intensity of scattering for even modest applied fields (tens of millitesla) when spin-flip scattering is appreciable; when the spin-flip cross section is small compared to the non-spin-flip, the corrections remain small.

This so-called Zeeman shift in spin-flipped reflected neutrons was first described by Felcher et al. (1995) and has been observed by many others (Felcher et al., 1996). Kozhevnikov et al. (2012) presented a clear description of the geometry of the incident and scattered beams. The reflectivity calculation formalism including the Zeeman term was briefly described by van de Kruijs et al. (2000), Fitzsimmon et al. (2006) and Liu et al. (2011), but to our knowledge a detailed description of the calculation is not available in the literature, nor has such a calculation been incorporated into commonly used modeling software.

These shifts are not a major concern in many experiments (Liu et al., 2011) because the effect is significant only when there is both a large applied field and strong spin-flip scattering. At low fields the corrections are minimal, and at high fields the magnetization tends to align parallel to the applied 
field, so there is insignificant spin-flip scattering. However, there are important cases where accounting for the Zeeman shift is necessary for appropriately measuring and analyzing data. A technologically relevant example is the study of highanisotropy magnetic materials used in advanced data-storage applications (Liu et al., 2011). In such cases the sample magnetization can be non-collinear even with large applied fields.

In this paper we will address the requirements for setting up a measurement in a large field in the case where the spin-flip scattering is not negligible. We present the changes that need to be made to an existing commonly used computer algorithm (implemented in gepore.f; Majkrzak et al., 2006) in order to calculate the scattering correctly, and we present recommended practices for performing the measurements when both the applied magnetic field $\mathbf{H}$ and the magnetization $\mathbf{M}$ are large and not parallel to each other. This implies a large magnetic anisotropy in the system. We take advantage of the large shape anisotropy in a thin film of a soft magnetic material in the example experiment section of this paper to show clearly the effects we are discussing.

We must also address the meaning of the word 'specular'. In many texts on reflectivity the definition is given that the angle of incidence equals the angle of reflection, or that the out-ofplane component of the momentum of the incoming beam $k_{z \text {,in }}$ is equal in magnitude to that of the outgoing reflected beam $k_{z, \text { out }}$. Here we will use a more functional definition based on the momentum transfer $\mathbf{Q} \equiv \mathbf{k}_{\text {in }}-\mathbf{k}_{\text {out }}$. We define the reflectivity as specular on the condition that the in-plane momentum transfers $Q_{x}=0$ and $Q_{y}=0$, so that the momentum transfer $\mathbf{Q} \equiv Q_{z} \hat{z}$ (perpendicular to the surface), as is expected when reflecting from planar layered samples.

As we will demonstrate, some of the kinetic energy along $\hat{z}$ is traded for potential energy during a spin-flip process, so the earlier definitions do not apply in this circumstance, while $\mathbf{Q}$ remains strictly out of plane.

\section{Boundary conditions}

Starting with the general Schrödinger equation for a neutron with spin $\frac{1}{2}$,

$$
\left[-\frac{\hbar^{2}}{2 m} \nabla^{2} \hat{1}+\hat{V}(\mathbf{r})-E \hat{1}\right]\left[\begin{array}{l}
\psi^{+}(\mathbf{r}) \\
\psi^{-}(\mathbf{r})
\end{array}\right]=0
$$

where $\psi^{ \pm}$is the spin-dependent wavefunction for the neutron,

$$
\hat{1}=\left(\begin{array}{ll}
1 & 0 \\
0 & 1
\end{array}\right)
$$

$\nabla^{2}$ is the Laplacian (spatial second derivative) and the hatted components indicate a Pauli spin matrix with $z^{\prime}$ as the quantization axis. We use the notation $z^{\prime}$ for coordinates in the spin quantization reference frame to distinguish it from the scattering geometric reference frame where $z$ is defined to be the surface normal direction for the planar sample, and there is no requirement that $\hat{z} \| z^{\prime}$. The potential of the particle is made up of a scalar nuclear potential $V_{\text {nuc }}$ and a magnetic potential due to the field $\mathbf{B}$ :

$$
\hat{V}=\mu_{\mathrm{N}} \boldsymbol{\sigma} \cdot \mathbf{B}+V_{\mathrm{nuc}} \hat{1}
$$

where

$$
\boldsymbol{\sigma} \cdot \mathbf{B}=\left(\begin{array}{cc}
0 & 1 \\
1 & 0
\end{array}\right) B_{x^{\prime}}+\left(\begin{array}{cc}
0 & -i \\
i & 0
\end{array}\right) B_{y^{\prime}}+\left(\begin{array}{cc}
1 & 0 \\
0 & -1
\end{array}\right) B_{z^{\prime}} .
$$

Here, $\mu_{\mathrm{N}}=5.0507835 \times 10^{-27} \mathrm{~J} \mathrm{~T}^{-1}$ is the nuclear magnetic moment.

In the 'prepared' spin-polarized beam, we define the direction of the guide field to be $\hat{z}^{\prime}$, so there are no off-diagonal elements to the potential above (because $B_{x^{\prime}} \equiv B_{y^{\prime}} \equiv 0$ ) and the equation decouples into two linear equations for potentials with $V=V_{\text {nuc }} \pm \mu_{\mathrm{N}} B_{z^{\prime}}$.

When the beam enters the fronting medium with nonnegligible $\mathbf{B}$, there is no physical restriction on the direction of B, but from an experimental design perspective we note that, if the magnetic field in the fronting medium is not parallel to the applied laboratory field direction $\left(\hat{z}^{\prime}\right)$, i.e. there is a nonzero $B_{x^{\prime}}$ or $B_{y^{\prime}}$ component to the field in this region, the wavefunction will be angularly split owing to the field-dependent difference between $k_{\mathrm{F}, x}^{+}$and $k_{\mathrm{F}, x}^{-}$. The mutual coherence of the two resulting beams will be impractical to calculate over the macroscopic distances the beam will then travel after being split.

This is not to be confused with the angular splitting which occurs as the beam interacts with the horizontal layers of the sample, which is what is usually being discussed when describing reflectivity, and which is fully taken into account in the calculations below.

Now, restricting ourselves to the case in which the $\mathbf{B}$ field in the fronting medium is parallel to the guide field outside the fronting medium, we can fully describe the interaction of the neutron with the sample as in Fig. 1.

The incident neutron is 'prepared' in either the $I^{+}$or $I^{-}$spin state using techniques described elsewhere (Dura et al., 2006; Majkrzak et al., 2006). Neglecting the contribution of a very small magnetic guide field, the total energy of both states is

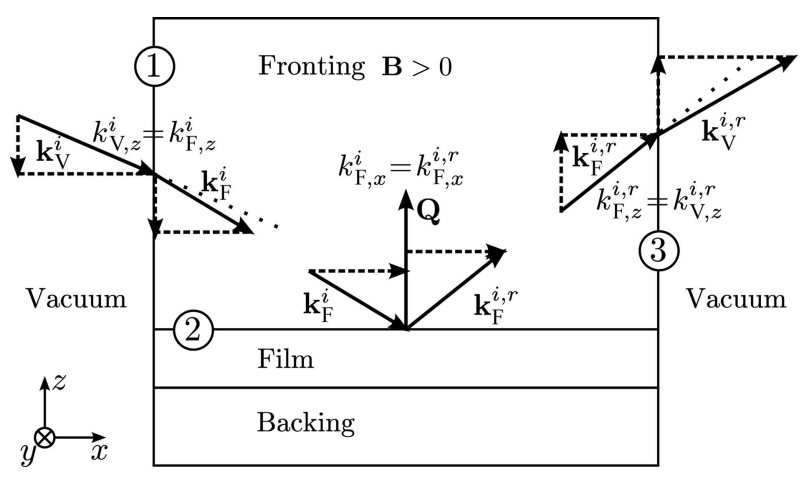

Figure 1

Diagram showing the various components of the $\mathbf{k}$ and $\mathbf{q}$ vectors for a polarized specularly reflected neutron entering a magnetic sample system from the left-hand side. The coordinate system is also shown. 
nearly the same for the same $\mathbf{k}_{\mathrm{V}}$ and is equal to the kinetic energy:

$$
E_{\mathrm{V}, x y z}^{+} \simeq E_{\mathrm{V}, x y z}^{-} \simeq \frac{\hbar^{2}}{2 m}\left(k_{\mathrm{V}, x}^{2}+k_{\mathrm{V}, y}^{2}+k_{\mathrm{V}, z}^{2}\right) .
$$

We note that the problem as defined has no $y$ dependence: there are no interfaces along that direction (out of the page of the figure) and so the solution for the wave equation along $y$ is for a plane wave $\exp \left(i k_{y} y\right)$ with constant kinetic energy that can be included in the total energy $E$. The problem can then be treated as a two-dimensional Schrödinger equation in $x$ and $z$, with

$$
E_{\mathrm{V}, x z}^{ \pm} \simeq \frac{\hbar^{2}}{2 m}\left(k_{\mathrm{V}, x}^{2}+k_{\mathrm{V}, z}^{2}\right)=E_{\mathrm{V}, x y z}^{ \pm}-\frac{\hbar^{2}}{2 m} k_{\mathrm{V}, y}^{2} .
$$

When the neutron enters the fronting medium at the boundary labeled 1 in Fig. 1, the potential energy changes in a spindependent way so that

$$
\begin{aligned}
& E_{\mathrm{F}, x z}^{+}=\frac{\hbar^{2}}{2 m}\left[\left(k_{\mathrm{F}, x}^{+}\right)^{2}+\left(k_{\mathrm{F}, z}^{+}\right)^{2}+4 \pi\left(\rho_{\mathrm{F}, \mathrm{N}}+\rho_{\mathrm{F}, B}\right)\right], \\
& E_{\mathrm{F}, x z}^{-}=\frac{\hbar^{2}}{2 m}\left[\left(k_{\mathrm{F}, x}^{-}\right)^{2}+\left(k_{\mathrm{F}, z}^{-}\right)^{2}+4 \pi\left(\rho_{\mathrm{F}, \mathrm{N}}-\rho_{\mathrm{F}, B}\right)\right],
\end{aligned}
$$

where the notation $\mathbf{k}_{\mathrm{F}, \mathrm{V}}^{i}$ indicates the wavevector in the medium (with the subscripts $\mathrm{F}$ and $\mathrm{V}$ denoting the fronting and the vacuum, respectively) with spin state $i(+$ or -$)$. The nuclear scattering length density (SLD) of the fronting medium $\rho_{\mathrm{F}, \mathrm{N}}$ depends on the isotopic composition of the medium, while $\rho_{\mathrm{F}, B}$ is the magnetic SLD, which can be calculated from the magnetic field in that layer by

$$
\rho_{B}=\frac{2 \mu_{\mathrm{n}} m_{\mathrm{n}}|\mathbf{B}|}{4 \pi \hbar^{2}} \simeq B \times 2.31605 \times 10^{-6} \stackrel{\circ}{\mathrm{A}}^{-2} \mathrm{~T}^{-1} .
$$

Here $\mu_{\mathrm{n}}$ and $m_{\mathrm{n}}$ are the magnetic moment and mass of a neutron, respectively, and $B$ is the magnitude of the magnetic field in the fronting medium (in tesla).

Since the magnetic field inside the vertical boundary is parallel to (though possibly much bigger than) the field outside, the $(+)$ or $(-)$ spin state inside the vertical boundary matches the prepared state. Also, by symmetry $k_{z}$ must be preserved across the vertical boundary labeled 1 , between the vacuum and the fronting medium, so $k_{\mathrm{V}, z}=k_{\mathrm{F}, z}$. Since $E_{\mathrm{V}, x z}^{+}=$ $E_{\mathrm{F}, x z}^{+}$as well, this means that

$$
\begin{aligned}
& \left(k_{\mathrm{F}, x}^{+}\right)^{2}=\left(k_{\mathrm{V}, x}\right)^{2}-4 \pi\left(\rho_{\mathrm{F}, \mathrm{N}}+\rho_{\mathrm{F}, B}\right), \\
& \left(k_{\mathrm{F}, x}^{-}\right)^{2}=\left(k_{\mathrm{V}, x}\right)^{2}-4 \pi\left(\rho_{\mathrm{F}, \mathrm{N}}-\rho_{\mathrm{F}, B}\right),
\end{aligned}
$$

which changes the angle of the neutron beam inside the fronting medium (this is refraction, as indicated by the shortened $k_{x}$ on the right-hand side of boundary 1 in Fig. 1). The energy trade in $k_{x}$ is reversed when the neutron exits the fronting at the boundary labeled 3 ; the $k_{\mathrm{V}, x}$ on the right is the same as that on the left. This is not in general true for $k_{\mathrm{V}, z}$, as we will see.

Now we consider the next set of boundaries in the problem, namely the horizontal interfaces of the sample under investigation. The first of these is the top interface labeled 2, between the fronting and the sample. When the neutron interacts with this structure, it is possible to have a spin-flip event, so we introduce a second indicator $r$ (for reflected) in the notation $\mathbf{k}_{\mathrm{F}}^{i, r}$ for the spin state of the outgoing neutron (still in the fronting medium). We retain the indicator $i$ for the incident neutron spin state because this determines the energy in the fronting, as described above.

We are considering the standard specular reflectometry case where the sample under investigation is homogenous in plane. Thus, while $k_{z}$ was conserved across boundary 1 , now $k_{x}$ is conserved across boundaries such as 2 , so

$$
\begin{aligned}
& k_{\mathrm{F}, x}^{+,-}=k_{\mathrm{F}, x}^{+,+}=k_{\mathrm{F}, x}^{+}, \\
& k_{\mathrm{F}, x}^{-,+}=k_{\mathrm{F}, x}^{-,-}=k_{\mathrm{F}, x}^{-},
\end{aligned}
$$

and because the total energy of the neutron is conserved during elastic scattering, we can write

$$
\begin{aligned}
E_{\mathrm{F}, x z}^{+,-} & =\frac{\hbar^{2}}{2 m}\left[\left(k_{\mathrm{F}, x}^{+,-}\right)^{2}+\left(k_{\mathrm{F}, z}^{+,-}\right)^{2}+4 \pi\left(\rho_{\mathrm{F}, \mathrm{N}}-\rho_{\mathrm{F}, B}\right)\right] \\
& =E_{\mathrm{F}, x z}^{+} .
\end{aligned}
$$

Subtracting this from equation (6) gives

$$
\left(k_{\mathrm{F}, z}^{+,-}\right)^{2}=\left(k_{\mathrm{F}, z}^{+}\right)^{2}+8 \pi \rho_{\mathrm{F}, B} .
$$

In a similar fashion for the (-) state, we can obtain

$$
\left(k_{\mathrm{F}, z}^{-,+}\right)^{2}=\left(k_{\mathrm{F}, z}^{-}\right)^{2}-8 \pi \rho_{\mathrm{F}, B},
$$

while the non-spin-flipped neutrons are not shifted:

$$
\begin{aligned}
& \left(k_{\mathrm{F}, z}^{+,+}\right)^{2}=\left(k_{\mathrm{F}, z}^{+}\right)^{2}, \\
& \left(k_{\mathrm{F}, z}^{-,-}\right)^{2}=\left(k_{\mathrm{F}, z}^{-}\right)^{2} .
\end{aligned}
$$

At the next boundary, labeled 3, where the neutrons exit the fronting material and go back into the laboratory environment (vacuum), $k_{z}$ is again conserved by symmetry, as it was at boundary 1 , so the shift in the spin-flipped $k_{z}$ is carried across this boundary $\left(k_{\mathrm{V}, z}^{i, r}=k_{\mathrm{F}, z}^{i, r}\right.$ for all $\left.i, r\right)$.

The difference between $k_{\mathrm{V}, z}^{+,-}$and $k_{\mathrm{V}, z}^{+,+}$leads to a different propagation direction for the spin-flipped neutron; this measurable angular shift is referred to as Zeeman splitting.

There are values of $k_{\mathrm{F}, z}^{-}$for which $\left(k_{\mathrm{F}, z}^{-}\right)^{2}<8 \pi \rho_{\mathrm{F}, B}$ and therefore the calculated momentum squared for the spinflipped reflection $\left(k_{\mathrm{F}, z}^{-,+}\right)^{2}$ is negative, so that $k_{\mathrm{F}, z}^{-,+}$becomes purely imaginary. The calculated amplitude for this reflection is valid at the interface, but this is an evanescent wave that decays as it moves away from the sample. The value of the measured reflectivity corresponds to the amplitude at the detector and is thus effectively zero in this case.

\subsection{Details of magnetic field geometry}

In the above discussion, the transition from vacuum with zero applied field to a high-field region (also with a possibly nonzero nuclear SLD) was described as a sharp boundary perpendicular to the sample plane (along $x$ ). In that case, the momentum along $z$ is unchanged by the transition, $k_{\mathrm{F}, z}=k_{\mathrm{V}, z}$, and energy conservation leads only to a change in $k_{x}:\left(k_{\mathrm{F}, x}^{+}\right)^{2}+$ $4 \pi\left(\rho_{\mathrm{F}, \mathrm{N}}+\rho_{\mathrm{F}, B}\right)=k_{\mathrm{V}, x}^{2}$. 
In real laboratory environments the magnetic field transition is not as abrupt as what is shown in Fig. 1, and the direction is not perfectly defined, though typically the applied magnetic field is realized in a small volume centered on the sample and the field gradient experienced by the probe neutron is, to first order, radial with respect to the sample. Since for any gradient in the potential the momentum components perpendicular to the gradient direction are conserved throughout the interaction with the potential, the abruptness of the transition is irrelevant and only the direction is important.

So, compared to a more realistic radial magnetic potential gradient parallel to the neutron momentum, we expect that, by using our simplified rectangular boundary conditions (where the sharp gradient at that boundary is along $\hat{x}$ and is nearly but not quite parallel to $\mathbf{k}_{\text {in }}$ ), we introduce an error in the calculated $\left(k_{\mathrm{F}, z}^{ \pm}\right)^{2}$ proportional to $\sin ^{2} \delta$, where $\delta$ is the angle between the normal to the rectangular boundary and $\mathbf{k}_{\text {in }}$. Because of the right angle between that boundary and the film surface, the result is that $\delta$ coincides with the incident angle $\theta_{\text {in }}$ of the neutron on the film surface.

At the small angles $\left(\theta_{\text {in }}<6^{\circ}\right)$ commonly seen for the incident angle during a reflectometry measurement, this results in a maximum correction to $\left(k_{\mathrm{F}, z}^{ \pm}\right)^{2}$ from the model proposed above of the order of $1 \%$ of $\pm 4 \pi \rho_{B}$ (with the opposite correction made to $\left.E_{\mathrm{F}, z}^{ \pm}\right)$. At the even smaller angles $\left(\theta_{\text {in }} \simeq\right.$ $0.5^{\circ}$ ) near the critical edge where these shifts might affect the modeling, the correction is just $0.01 \%$ of the magnetic SLD. For this reason, in many cases it is a reasonable approximation that all of the kinetic energy shift in the fronting region prior to the sample is along the $x$ direction, as defined by the sample coordinate system in Fig. 1.

\section{Calculation of the spin-dependent reflectivity}

\subsection{One-dimensional Schrödinger equation}

Again considering the region between boundaries 1 and 3 as above, we can calculate the reflectivity of the horizontally layered structure there by reducing the Schrödinger equation to a single spatial dimension $z$ and solving with the boundary conditions laid out above. Since the potential is constant as a function of $x$ in this region (as it is for $y$ everywhere), and $V(\mathbf{r})=V(z)$, the one-dimensional spin-dependent Schrödinger equation for the neutron is then (Majkrzak et al., 2006)

$$
\left[-\frac{\hbar^{2}}{2 m} \frac{\partial^{2}}{\partial z^{2}} \hat{1}+\hat{V}(z)-E_{\mathrm{F}, z}^{i} \hat{1}\right]\left[\begin{array}{c}
\psi^{i,+}(z) \\
\psi^{i,-}(z)
\end{array}\right]=0,
$$

where

$$
\begin{aligned}
\hat{V}(z) & =\left[\begin{array}{cc}
V^{++}(z) & V^{+-}(z) \\
V^{-+}(z) & V^{--}(z)
\end{array}\right] \\
& =\frac{4 \pi \hbar^{2}}{2 m}\left(\begin{array}{cc}
\rho_{\mathrm{N}}+\rho_{B z^{\prime}} & \rho_{B x^{\prime}}-i \rho_{B y^{\prime}} \\
\rho_{B x^{\prime}}+i \rho_{B y^{\prime}} & \rho_{\mathrm{N}}-\rho_{B z^{\prime}}
\end{array}\right)(z),
\end{aligned}
$$

and we fold the constant kinetic energy along $x$ into $E$ as we did for $y$ before:

$$
E_{\mathrm{F}, z}^{i}=E_{\mathrm{F}, x z}^{i}-\frac{\hbar^{2}}{2 m} k_{\mathrm{F}, x}^{2} .
$$

$E_{\mathrm{F}, z}^{i}$ depends on the spin state of the incident neutron as well as on the potential in the fronting medium, as

$$
E_{\mathrm{F}, z}^{ \pm}=\frac{\hbar^{2}}{2 m}\left[4 \pi\left(\rho_{\mathrm{F}, \mathrm{N}} \pm \rho_{\mathrm{F}, B}\right)+\left(k_{\mathrm{V}, z}\right)^{2}\right] .
$$

A set of solutions to equation 15 is laid out in the paper by Majkrzak et al. (2006), as (except now keeping track of the polarization $i$ of the incident state)

$$
\begin{aligned}
\psi^{i,+}(z) & =\sum_{j=1}^{4} C_{j}^{i} \exp \left(S_{j}^{i} z\right), \\
\psi^{i,-}(z) & =\sum_{j=1}^{4} \mu_{j} C_{j}^{i} \exp \left(S_{j}^{i} z\right),
\end{aligned}
$$

where

$$
\begin{aligned}
& S_{1}^{i}=\left[4 \pi\left(\rho_{\mathrm{N}}+\rho_{B}\right)-\frac{2 m}{\hbar^{2}} E_{\mathrm{F}, z}^{i}\right]^{1 / 2}, \\
& S_{2}^{i}=-S_{1}^{i}, \\
& S_{3}^{i}=\left[4 \pi\left(\rho_{\mathrm{N}}-\rho_{B}\right)-\frac{2 m}{\hbar^{2}} E_{\mathrm{F}, z}^{i}\right]^{1 / 2}, \\
& S_{4}^{i}=-S_{3}^{i},
\end{aligned}
$$

and

$$
\begin{aligned}
& \mu_{1}=\mu_{2}=\frac{B+B_{x^{\prime}}+i B_{y^{\prime}}-B_{z^{\prime}}}{B+B_{x^{\prime}}-i B_{y^{\prime}}+B_{z^{\prime}}} \\
& \mu_{3}=\mu_{4}=\frac{-B+B_{x^{\prime}}+i B_{y^{\prime}}-B_{z^{\prime}}}{-B+B_{x^{\prime}}-i B_{y^{\prime}}+B_{z^{\prime}}},
\end{aligned}
$$

and the terms $C_{j}^{i}$ are the complex coefficients of the four components.

Within the fronting medium $\mathrm{F}$ the propagation constants $S$ are simply equal to the incident wave value $i k_{\mathrm{F}, z}$, since the potentials $\rho$ cancel between equations (18) and (20) for the incident beams $I^{+}, I^{-}$.

When the external magnetic potential is negligible, the $E$ in the above equations is the same for both incident beam polarizations but, in general, $E_{\mathrm{F}, z}^{+} \neq E_{\mathrm{F}, z}^{-}$for a sufficiently large field. Because of this, if we measure the reflectivity at the same $k_{\mathrm{V}, z}$ for both the $I^{+}$and $I^{-}$states, we have to distinguish between polarization states for the incoming beam.

This distinction based on the Zeeman energy of the neutron in the fronting medium is the basis for a small but critical change to the existing computer code for calculating reflectivity (see gepore.f; Majkrzak et al., 2006). There the term proportional to $E$ is set to $Q^{2} / 4-4 \pi \rho_{\mathrm{F}, \mathrm{N}}$ (for $Q \equiv 2 k_{\mathrm{V}, z}$ ), which accounts for only the kinetic and nuclear potential energy in the fronting medium; this gives the correct answer for any case except when the Zeeman term is appreciable, so we will use $\left(2 m / h^{2}\right) E_{\mathrm{F}, z}^{ \pm}$instead, which includes the kinetic, nuclear and magnetic energies in the fronting medium appropriate for the relevant incident spin state.

Also in the previous code, equation (21) for the ratio of $\psi^{-}$ to $\psi^{+}$components is replaced with 


$$
\begin{aligned}
& \mu_{1}=\mu_{2}=\exp \left(i \theta_{\mathbf{M}}\right), \\
& \mu_{3}=\mu_{4}=-\exp \left(i \theta_{\mathbf{M}}\right),
\end{aligned}
$$

where $\theta_{\mathbf{M}}$ is the in-plane $(x, y)$ angle, with the underlying implicit assumptions that the contribution to $\mathbf{B}$ from $\mathbf{H}_{\text {applied }}$ is negligible and that the net $B_{z}$ (out of the sample plane) is zero. These assumptions are quite reasonable for low values of $\mathbf{H}$ even when there is a large perpendicular magnetization, because for thin-film samples the demagnetization field $\left|\mathbf{H}_{\mathrm{D}}\right|=$ $H_{\mathrm{D} z} \simeq-M_{z}$ almost completely cancels the contribution of the net perpendicular component $M_{z}$ to $B_{z}$ [because $\mathbf{B}=\mu_{0}(\mathbf{M}+$ $\left.\mathbf{H}_{\text {applied }}+\mathbf{H}_{\mathrm{D}}+\cdots\right)$ ]. [Of course, the demagnetizing field does not exactly cancel the magnetic field along $z$, and there is a non-zero $\mathbf{B}$ field at large distances from the sample (measurable with a magnetometer) which is proportional to the volume integral of $\mathbf{M}$. In the thin-film geometry, the surface to volume ratio goes to infinity, and this is why there is effectively zero $B_{\perp}$ at the surface.]

Now that we are including the effects of an arbitrary external field, however, we must include $B_{z} \simeq H_{z}$ and return the more general equation (21) for $\mu$.

Since the applied field along $z$ and the associated potential are constant across the sample volume, this does not lead to any additional scattering, which in the continuum limit happens only at discontinuities in the potential. Still, it must be included since it affects (or rather, effects) the relative phase of spin-flipped versus non-spin-flipped portions of the neutron wavefunction, which changes the measured reflectivity.

\subsection{Reparametrization of $\psi$ and reflectivity derivation}

In the more general equation (21), the values of $\mu_{1}$ or $\mu_{3}$ become unbounded when $\mathbf{B}$ approaches a direction perfectly parallel or antiparallel to the spin quantization direction $\hat{z}^{\prime}$. This situation of course always occurs in the fronting (and backing) medium, since there the field direction defines the quantization direction, $\hat{z}^{\prime} \equiv \hat{B}_{\mathrm{F}}$. While the equations are analytically correct when one takes the appropriate limits, floating-point computation errors are introduced when multiplying and dividing arbitrarily large numbers in a computer.

Since the $\mu$ values in equation (19) serve only to describe the ratio between the components of $\psi^{+}$and $\psi^{-}$, and because $\mu_{1}=\mu_{2}$ and $\mu_{3}=\mu_{4}$, we can rearrange that equation as

$$
\begin{aligned}
\psi^{i,+}(z)= & D_{1}^{i} \exp \left(S_{1}^{i} z\right)+D_{2}^{i} \exp \left(S_{2}^{i} z\right)+\gamma D_{3}^{i} \exp \left(S_{3}^{i} z\right) \\
& +\gamma D_{4}^{i} \exp \left(S_{4}^{i} z\right) \\
\psi^{i,-}(z)= & \beta D_{1}^{i} \exp \left(S_{1}^{i} z\right)+\beta D_{2}^{i} \exp \left(S_{2}^{i} z\right)+D_{3}^{i} \exp \left(S_{3}^{i} z\right) \\
& +D_{4}^{i} \exp \left(S_{4}^{i} z\right)
\end{aligned}
$$

and, relating these constants to our previous parametrization, we obtain

$$
\begin{aligned}
\beta & =\mu_{1}, \\
\gamma & =1 / \mu_{3}, \\
D_{1} & =C_{1}, \\
D_{2} & =C_{2}, \\
D_{3} & =C_{3} / \gamma, \\
D_{4} & =C_{4} / \gamma .
\end{aligned}
$$

This solution to the Schrödinger equation is valid within any layer of the material, so we can calculate the reflectivity by using the boundary conditions to stitch together solutions from adjacent layers. At any interface, the value of the wavefunction and its first derivative $\left[\psi, \psi^{\prime}\right]$ must be continuous across that boundary. We can write the wavefunction in terms of the $D_{j}^{i}$ coefficients in that layer (for either incident spin state $i$ ):

$$
\left[\begin{array}{c}
\psi^{i,+}(z) \\
\psi^{i,-}(z) \\
\psi^{\prime i,+}(z) \\
\psi^{\prime i,-}(z)
\end{array}\right]=\chi(z)\left(\begin{array}{c}
D_{1}^{i} \\
D_{2}^{i} \\
D_{3}^{i} \\
D_{4}^{i}
\end{array}\right)
$$

where from equation (23)

$$
\begin{aligned}
& \chi(z)=\left(\begin{array}{cccc}
1 & 1 & \gamma & \gamma \\
\beta & \beta & 1 & 1 \\
S_{1} & -S_{1} & \gamma & -\gamma \\
\beta S_{1} & -\beta S_{1} & S_{3} & -S_{3}
\end{array}\right) \\
& \times\left[\begin{array}{cccc}
\exp \left(S_{1} z\right) & 0 & 0 & 0 \\
0 & \exp \left(-S_{1} z\right) & 0 & 0 \\
0 & 0 & \exp \left(S_{3} z\right) & 0 \\
0 & 0 & 0 & \exp \left(-S_{3} z\right)
\end{array}\right] \\
& =\left(\begin{array}{cccc}
1 & 1 & \gamma & \gamma \\
\beta & \beta & 1 & 1 \\
S_{1} & -S_{1} & \gamma & -\gamma \\
\beta S_{1} & -\beta S_{1} & S_{3} & -S_{3}
\end{array}\right)[\exp (\mathbf{S} z) \cdot \mathbf{I}] \text {. }
\end{aligned}
$$

Here $\gamma, \beta$ and $\mathbf{S}$ are specific to the layer $l$ and incident spin state $i$ being calculated. At the boundary between layers $l$ and $l+1$ (we will define the boundary position $z \equiv Z_{l}$ here), we have $\psi_{l}=\psi_{l+1}$ and $\psi_{l}^{\prime}=\psi_{l+1}^{\prime}$, so that

$$
\chi_{l}\left(Z_{l}\right)\left(\begin{array}{c}
D_{1, l} \\
D_{2, l} \\
D_{3, l} \\
D_{4, l}
\end{array}\right)=\chi_{l+1}\left(Z_{l}\right)\left(\begin{array}{c}
D_{1, l+1} \\
D_{2, l+1} \\
D_{3, l+1} \\
D_{4, l+1}
\end{array}\right)
$$

Thus to get $\left\{D_{l+1}\right\}$ from $\left\{D_{l}\right\}$, we invert $\chi_{l+1}$ and

$$
\chi_{l+1}^{-1}\left(Z_{l}\right) \chi_{l}\left(Z_{l}\right)\left(\begin{array}{c}
D_{1, l} \\
D_{2, l} \\
D_{3, l} \\
D_{4, l}
\end{array}\right)=\left(\begin{array}{c}
D_{1, l+1} \\
D_{2, l+1} \\
D_{3, l+1} \\
D_{4, l+1}
\end{array}\right),
$$




$$
\chi^{-1}(z)=\frac{1}{2(1-\gamma \beta)}[\exp (-\mathbf{S} z) \cdot \mathbf{I}]\left(\begin{array}{cccc}
1 & -\gamma & \frac{1}{S_{1}} & \frac{-\gamma}{S_{1}} \\
1 & -\gamma & \frac{-1}{S_{1}} & \frac{\gamma}{S_{1}} \\
-\beta & 1 & \frac{-\beta}{S_{3}} & \frac{1}{S_{3}} \\
-\beta & 1 & \frac{\beta}{S_{3}} & \frac{-1}{S_{3}}
\end{array}\right)
$$

Since $\gamma$ and $\beta$ never have the same complex phase, the denominator of equation (29) is never zero. Then for a structure with $N$ layers, the coefficients of the transmitted wave $\left\{D_{j, N}^{i}\right\}$ are related to the coefficients in the incident medium $\left\{D_{j, 0}^{i}\right\}$ by

$$
\begin{aligned}
\left(\begin{array}{c}
D_{1, N}^{i} \\
D_{2, N}^{i} \\
D_{3, N}^{i} \\
D_{4, N}^{i}
\end{array}\right) & =\prod_{N}^{1}\left(\chi_{n}^{i}\right)^{-1}\left(Z_{n-1}\right) \chi_{n-1}^{i}\left(Z_{n-1}\right)\left(\begin{array}{c}
D_{1,0}^{i} \\
D_{2,0}^{i} \\
D_{3,0}^{i} \\
D_{4,0}^{i}
\end{array}\right) \\
& =B^{i}\left(\begin{array}{c}
D_{1,0}^{i} \\
D_{2,0}^{i} \\
D_{3,0}^{i} \\
D_{4,0}^{i}
\end{array}\right)
\end{aligned}
$$

where the pairs of $\chi_{n}^{-1}\left(Z_{n-1}\right) \chi_{n-1}\left(Z_{n-1}\right)$ are $4 \times 4$ matrices. Note that the matrices differ for the different incident spin states, and so we have to calculate the matrix products $B^{+}$and $B^{-}$separately. The remaining boundary conditions are met by identifying the coefficients in the fronting medium for the two polarized incident states $I^{+}$and $I^{-}$,

$$
\left(\begin{array}{c}
D_{1,0} \\
D_{2,0} \\
D_{3,0} \\
D_{4,0}
\end{array}\right)^{+}=\left(\begin{array}{c}
I^{+} \\
r^{+,+} \\
0 \\
r^{+,-}
\end{array}\right) \text {and }\left(\begin{array}{c}
D_{1,0} \\
D_{2,0} \\
D_{3,0} \\
D_{4,0}
\end{array}\right)^{-}=\left(\begin{array}{c}
0 \\
r^{-,+} \\
I^{-} \\
r^{-,-}
\end{array}\right) \text {, }
$$

and the coefficients in the backing medium,

$$
\left(\begin{array}{c}
D_{1, N} \\
D_{2, N} \\
D_{3, N} \\
D_{4, N}
\end{array}\right)^{+}=\left(\begin{array}{c}
t^{+,+} \\
0 \\
t^{+,-} \\
0
\end{array}\right) \text { and }\left(\begin{array}{c}
D_{1, N} \\
D_{2, N} \\
D_{3, N} \\
D_{4, N}
\end{array}\right)^{-}=\left(\begin{array}{c}
t^{-,+} \\
0 \\
t^{-,-} \\
0
\end{array}\right) .
$$

Note that $D_{2, N}$ and $D_{4, N}$ are zero because of the boundary condition that the upward-traveling wave coefficient in the backing medium is zero (only downward-traveling waves corresponding to transmission are physical in our experimental setup).

For the $I^{+}$incident state, $I^{-} \equiv 0$ and vice versa, so we can calculate the ratios $r^{+,+} \equiv r^{+} / I^{+}, r^{+,-} \equiv r^{-} / I^{+}$etc. from the $B$ matrix product of equation (30) by using the zeros in $D_{2, N}$, $D_{4, N}$, which gives two equations with two unknowns $\left(r^{+}, r^{-}\right)$if we take the incident intensity to be unity. This gives for the different cross sections

$$
\begin{aligned}
r^{+,+} & =\frac{B_{24}^{+} B_{41}^{+}-B_{21}^{+} B_{44}^{+}}{B_{44}^{+} B_{22}^{+}-B_{24}^{+} B_{42}^{+}}, \\
r^{+,-} & =\frac{B_{21}^{+} B_{42}^{+}-B_{41}^{+} B_{22}^{+}}{B_{44}^{+} B_{22}^{+}-B_{24}^{+} B_{42}^{+}}, \\
r^{-,+} & =\frac{B_{24}^{-} B_{43}^{-}-B_{23}^{-} B_{44}^{-}}{B_{44}^{-} B_{22}^{-}-B_{24}^{-} B_{42}^{-}}, \\
r^{-,-} & =\frac{B_{23}^{-} B_{42}^{-}-B_{43}^{-} B_{22}^{-}}{B_{44}^{-} B_{22}^{-}-B_{24}^{-} B_{42}^{-}} .
\end{aligned}
$$

As can been seen in equation (26) above, the new constants $\gamma$ and $\beta$ have real physical significance as the mixing terms between $\psi^{+}$and $\psi^{-}$in a given layer, and for any $B_{z^{\prime}} \geq 0$ the constants $\gamma$ and $\beta$ are found inside the unit circle in the complex plane, i.e. $|\gamma, \beta| \leq 1$. In the fronting and backing media, they are both identically zero.

For a layer perfectly antiparallel to $\hat{z}^{\prime}, \beta$ and $\gamma$ will still be unbounded, but we further note that the numbering of the roots in equation (20) is arbitrary, so for every layer where $B_{z^{\prime}}<0$ we perform this switch for the matrix corresponding to that layer: $S_{1}^{\prime} \rightarrow S_{3}, S_{3}^{\prime} \rightarrow S_{1}, \gamma^{\prime} \rightarrow 1 / \beta$ and $\beta^{\prime} \rightarrow 1 / \gamma$. The new $\beta^{\prime}$ and $\gamma^{\prime}$ again have a magnitude less than or equal to one, and we can carry on with the calculation. This has no effect on the calculated reflectivity and the matrices are now all well conditioned (the magnitude of the matrix elements is always less than or equal to one). However, if the calculated values of $D_{j}$ are to be used to reconstruct the full wavefunction within that layer (say, for a distorted-wave Born approximation calculation) one has to be aware of the switch that was made, so that the multiplier $D_{j}$ is correctly associated with the propagation vector $S_{j}^{\prime}$ instead of $S_{j}$. As in the parallel case, for perfectly antiparallel $\mathbf{B}$ the mixing terms are exactly zero.

It is interesting that, in this new parametrization, the degenerate case where the magnetization is always aligned parallel or antiparallel to the applied $\mathbf{H}$ reduces very obviously to two uncoupled equations for the propagation of $\psi^{+}$and $\psi^{-}$, since the mixing terms in every layer are zero.

Since the spin of the incoming beam is never flipped in this case, the reference energy (including a Zeeman term) for the reflected neutron in the fronting medium will match the energy of the incident neutron for both possible incident spin states, and it can be subtracted from all the equations with no effect as an arbitrary energy offset. Thus, a Zeeman correction to the expected reflectivity will only be needed when there is non-collinear magnetization of the layers, but when this correction has to be made it will alter all the cross sections, including the non-spin-flip reflectivity (because of cross terms in the calculation between spin-flip and non-spin-flip reflectivity).

\subsection{Parametrization of $k$ and $E$}

The wave propagation constants $S$ in equation (20) are dependent only on the fixed potentials $\rho_{B}$ and $\rho_{\mathrm{N}}$ for that layer, and an energy term which depends on the spin state and $k_{z}$ of the incident neutron. If the reflectivity is solved for a given $E$, this corresponds to a set of $k_{\mathrm{F}, z}^{+} \neq k_{\mathrm{F}, z}^{-}$: 


$$
k_{\mathrm{F}, z}^{ \pm}=\left[\frac{2 m}{\hbar^{2}} E-4 \pi\left(\rho_{\mathrm{F}, \mathrm{N}} \pm \rho_{\mathrm{F}, B}\right)\right]^{1 / 2} .
$$

While this saves roughly a factor of two in computation time by mapping a single energy to the corresponding $k$ for the two incident spin states, it does not match the way a reflectometry experiment is typically carried out at a reactor-based beamline, where all four spin-dependent cross sections are measured for a single incident wavevector. A more natural instrument coordinate system is based on the incident and reflected angles $\left(\theta_{\text {in }}, \theta_{\text {out }}\right)$, which map onto $\left(k_{\text {in }}, k_{\text {out }}\right)$, and so we calculate the reflectivity twice for each value of $k_{\text {in }}$, once for each spin state and the corresponding value of $E_{\mathrm{F}, z}^{i}$.

\section{Measurement setup}

\subsection{Sample and detector angles}

While a shift in the reference potential had a large effect on the calculated reflectivities above, it is the angular shift (i.e. $\left.\theta_{\text {out }}-\theta_{\text {in }}\right)$ in the spin-flipped reflected beam that most affects the instrument setup for this type of measurement.

From the shift in $k_{z}$ in equations (12) and (13), we can calculate the outgoing angle of the reflected beam by

$$
\theta_{\text {out }}=\arctan \left[k_{z(\text { out })} / k_{x}\right] \text {. }
$$

From equation (35), it is easy to see that the angular shift of the spin-flipped reflected beam changes during the measurement, so a position-sensitive neutron detector will clearly facilitate experiments when the Zeeman effect is significant. However, some existing reactor-based polarized neutron reflectivity beamlines use pencil detectors. These have their own advantage of a very high detection efficiency, but an unconventional experimental procedure is required to take care of the Zeeman effect. Below, we detail the experimental setup using a pencil detector when the Zeeman effect is significant. For the four possible spin cross sections, three different values of $k_{z \text { (out) }}$ (and therefore of detector angle) are found for a single $k_{z(\mathrm{in})}$ in the specular condition $\left[k_{x(\mathrm{in})}=\right.$ $\left.k_{x(\text { out })}\right]$; one spin-flipped state is shifted higher and the other is shifted lower, while the two non-spin-flip processes give $k_{z \text { (in) }}=$ $k_{z \text { (out) }}$, so that $\theta_{\text {in }}=\theta_{\text {out }}$. One could just as well choose a fixed $\theta_{\text {out }}$ and $k_{z \text { (out) }}$, and calculate the three possible values of $k_{z \text { (in) }}$ for specular scattering, but for this discussion we will use $k_{z \text { (in) }}$ as the fixed quantity.

Since the polarization efficiency of the measurement system typically depends on the instrument geometry, for each of the three $\theta_{\text {out }}$ corresponding to a specularly reflected beam, all four spin cross sections have to be measured in order to extract an efficiency-corrected reflectivity for that angle. Only one of the corrected reflectivities out of the four will be used from the measurements at Zeeman-shifted angles $\theta_{\text {out }}^{-+}$and $\theta_{\text {out }}^{+-}$, while two reflectivities can be extracted from the nonspin-flip $\theta_{\text {out }}^{++}=\theta_{\text {out }}^{--}=\theta_{\text {in }}$. Overall, this increases the measurement time by a factor of three compared with an experiment without Zeeman corrections.

\section{Example measurement}

5.1. In-plane magnetic sample

In order to realize a large moment non-collinear with the field, a sample of a very magnetically soft material ( $\mathrm{Ni}-\mathrm{Fe}$ alloy) was grown on a single-crystal Si substrate and capped with a layer of Pd to prevent oxidation (as shown in Fig. 2).

For the principal polarized neutron reflectometry measurement of this study, an external magnetic field was applied for a measurement at a small angle to the film surface normal, as seen in Fig. 2. The demagnetizing field (shape anisotropy) of the film acts to keep the magnetization in plane, and for appropriate choices of field strength and angle this dominates over the torque from the applied field, so that the magnetization remains largely in plane. At the same time, the small in-plane component of the field $H_{x}$ is enough to align the layer into a single domain, pointing mostly along $x$.

This arrangement provides an ideal test of the equations, since there is both a large moment $\mathbf{M} \perp \mathbf{H}$ providing spin-flip scattering, and simultaneously a large $\mathbf{H}$ field which causes Zeeman splitting of the spin-flipped neutrons.

Using a vibrating-sample magnetometer measurement, we verified that the test sample is indeed magnetically soft with a saturation field in the hard (out-of-plane) direction of about $0.5 \mathrm{~T}$, and at $0.244 \mathrm{~T}$ (the applied field for the neutron measurements) the out-of-plane loop is linear with field, suggesting a coherent rotation. This verifies that it is a magnetically soft film with the expected shape anisotropy and no significant domain formation under the neutron measurement conditions.

A supplementary reflectometry measurement of the same sample was done in an in-plane saturating field in order to obtain a good value of the saturation magnetization of the soft magnetic layer. The scattering results from this measurement (not shown) are easily fitted to standard models of polarized neutron reflectometry without Zeeman corrections and indicate a saturation internal $B$ field of $0.551 \mathrm{~T}(M=$ $439.53 \mathrm{kA} \mathrm{m}^{-1}$ ). This is below the expected value for an $\mathrm{Ni}-\mathrm{Fe}$ alloy, which may result from the incorporation of oxygen in the film due to a poor vacuum during the deposition process. Nevertheless, for the purposes of this investigation, all that is

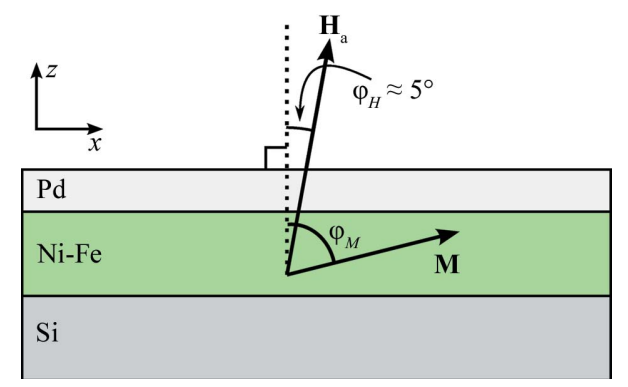

Figure 2

A sketch of the test sample, showing a side view of the layer structure of Pd $(200 \AA)$ on an Ni-Fe alloy $(600 \AA)$ on an Si substrate. The sample lateral size is $25 \times 25 \mathrm{~mm}$. The external applied field is slightly tilted with respect to the surface normal. 
required is a magnetically soft film, and the exact magnetization is irrelevant.

\subsection{Results}

The reflectivity measurements were undertaken at the Polarized Beam Reflectometer instrument (PBR) at the NIST Center for Neutron Research, with a supermirror spin polarizer and analyzer and current-coil Mezei-type spin flippers for the incident and reflected beams. In an applied field $\mu_{0} H_{\mathrm{a}}=$ $244 \mathrm{mT}$ at an angle as shown in Fig. 2, for a series of $k_{z \text { (in) }}$, all four spin cross sections were measured at each of the three outgoing angles corresponding to $\left(k_{z, \mathrm{~F}}^{+-}\right),\left(k_{z, \mathrm{~F}}^{+-}\right)$and $\left(k_{z, \mathrm{~F}}^{++}, k_{z, \mathrm{~F}}^{--}\right)$. The data for each of those outgoing angles were corrected for polarization and the relevant cross sections were extracted.

In Fig. 3 we show best fits to the data performed using the freely available Refl1D software package (Kirby et al., 2012; Kienzle et al., 2015), but without making corrections for the Zeeman effect. The symbols represent data points with error bars, and the lines represent the best fits possible.

We compare these with fits performed using a modification of the software, which includes the changes to the theory described in the first part of this manuscript. Both the data and the fits are presented in Fig. 4.

In the uncorrected fits in Fig. 3, we can clearly see that the splitting between the non-spin-flip scattering at low $k_{z \text { (in) }}$ is grossly underestimated in the best-fitting model. In this region the error bars are small, owing to the strong scattering, and this is what leads to the large minimum $\chi^{2}$ value of 25.0 for these fits. An enlargement of this region for comparing corrected versus uncorrected fits is shown in Fig. 5.

By contrast, the Zeeman-corrected fits are very good, with a $\chi^{2}$ value of 3.7. The visible deviations of the spin-flip data from

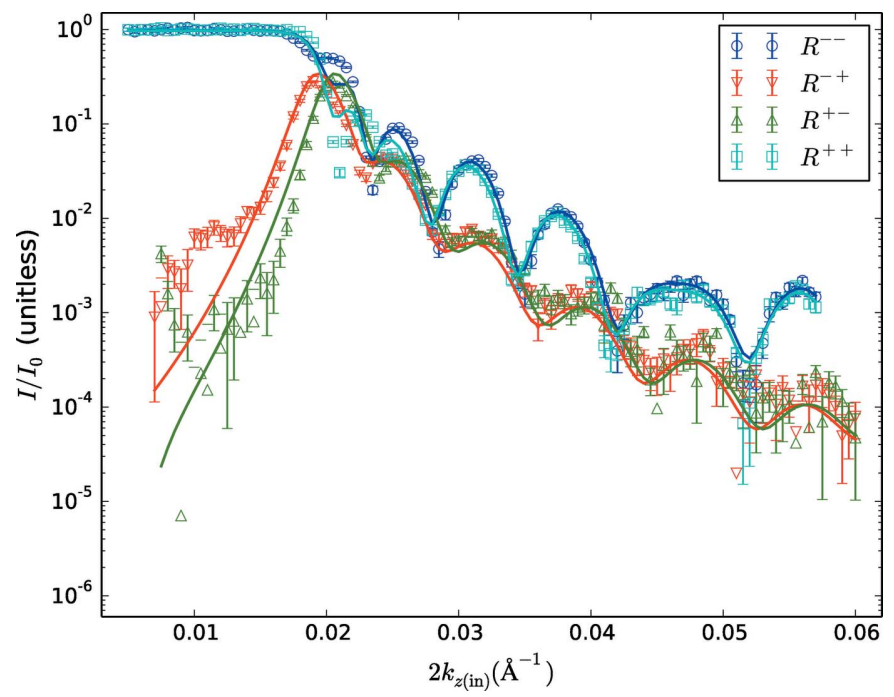

Figure 3

The reflectivity of the test sample and the best fit without including the effects of the Zeeman energy. The data are shown as open symbols, with error bars corresponding to $\pm 1 \sigma$ according to the counting statistics and the resolution function of the instrument. The fits are shown as solid lines (the reduced $\chi^{2}$ for these fits is 25.0). The data were parametrized and fitted according to $k_{z(\text { in) }}$. the fits at very low $k_{z \text { (in) }}$ are likely to be due to issues with the polarization correction (the correction is of the same magnitude as the spin-flip data there), and this does not significantly affect the rest of the fit. In the enlarged plot in Fig. 5(b), these fits clearly reproduce the data near the critical edge. The best fits to the data correspond to a magnetic SLD in the Ni-Fe layer of $\rho_{B}=1.12 \times 10^{-6} \AA^{-2}$ and thus $M_{x}=385 \mathrm{kA} \mathrm{m}^{-1}$.

The SLD profiles (nuclear and magnetic) resulting from the uncorrected fits in Fig. 3 are shown in Fig. 6 as dotted lines, while the SLD profiles from the corrected fits in Fig. 4 are shown as solid lines. The difference between the profiles is most significant in the region of the capping layer, where the

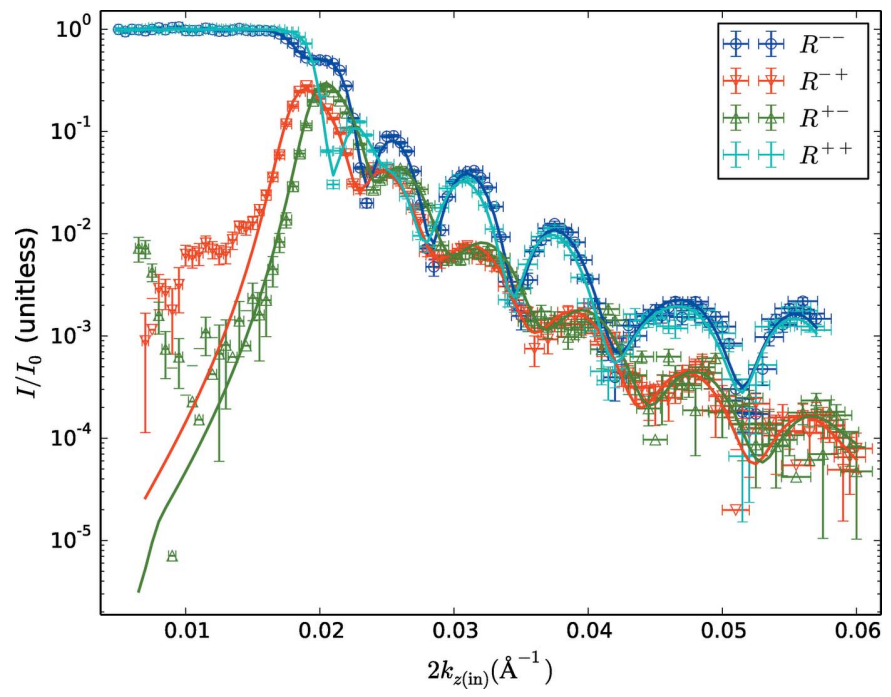

Figure 4

The reflectivity of the test sample, in all four cross sections, including fits. The data are shown as open symbols, with error bars corresponding to $\pm 1 \sigma$ according to the counting statistics and the resolution function of the instrument. The fits are shown as solid lines (the reduced $\chi^{2}$ for these fits is 3.7). The data were parametrized and fitted according to $k_{z \text { (in). }}$.

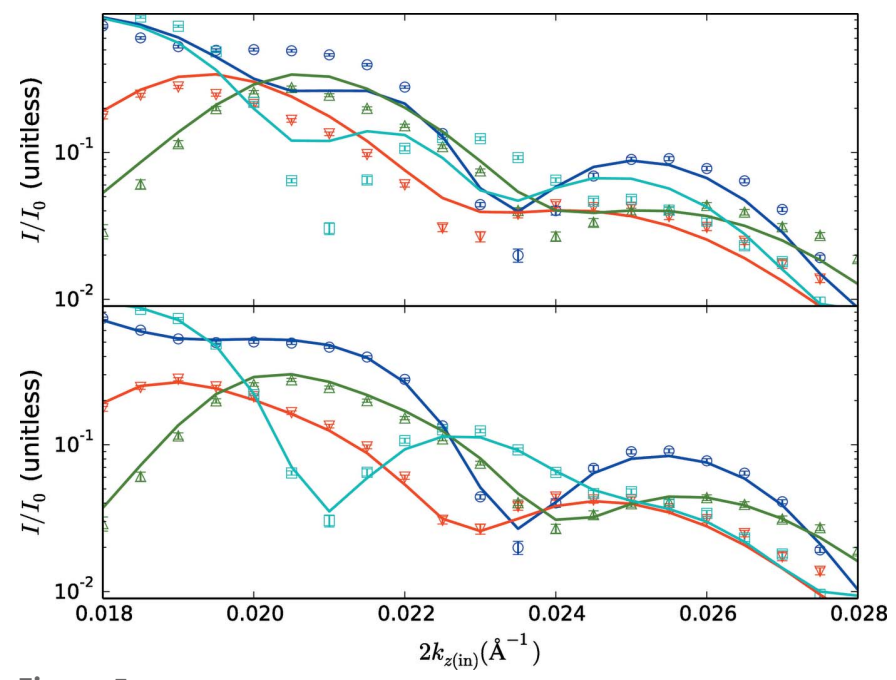

Figure 5

Enlargements of the reflectivity fits near the critical edge. (a) Enlargement corresponding to the fits without Zeeman corrections in Fig. 3. (b) Enlargement corresponding to the corrected fits in Fig. 4. A clear improvement in the quality of the fits is seen. Symbols and lines have the same meanings in this plot as in the originals. 


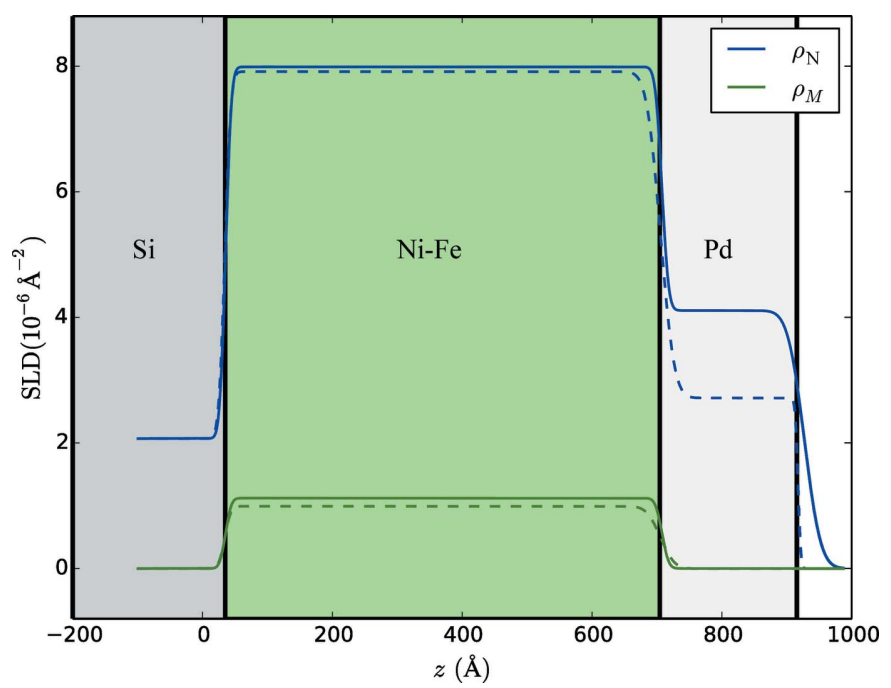

Figure 6

Scattering length density (SLD) profile corresponding to the fits shown in Fig. 4 (solid line) and Fig. 3 (dashed line). $\rho_{\mathrm{N}}$ and $\rho_{\mathrm{M}}$ refer to the nuclear and magnetic SLDs, respectively, in blue and green. For reference, the profiles are overlaid on a color-coded diagram of the sample structure as shown in Fig. 2.

uncorrected fit gives an unphysically low value of the nuclear SLD of the Pd capping layer $\left(2.7 \times 10^{-6} \AA^{-2}\right.$ rather than the expected value of $4.1 \times 10^{-6} \AA^{-2}$ ), and an unrealistically low roughness for the top interface, which one would expect to have a similar roughness to that of the interface immediately below.

The out-of-plane component of $\mathbf{M}$ for a system with uniaxial anisotropy arising from the demagnetization field is expected to be linearly dependent (when rotating coherently across the entire sample) on an applied out-of-plane field, reaching its saturation value at $H_{k}=M_{\mathrm{S}}$ (where $M_{\mathrm{S}}$ is the saturation magnetization). In our case, $M_{z} \simeq\left(H_{\mathrm{a}} / H_{k}\right) M_{\mathrm{S}}=(0.244 /$ $0.551) M_{\mathrm{S}}$, and since $M_{z}=M_{\mathrm{S}} \cos \varphi$ and $M_{x}=M_{\mathrm{S}} \sin \varphi$, we can extract an expected value for the in-plane magnetization $M_{x} \simeq$ $394 \mathrm{kA} \mathrm{m}^{-1}$, which agrees well with the fitted value of $385 \mathrm{kA} \mathrm{m}^{-1}$.

The most striking feature of the scattering in Fig. 4 is the large splitting between the non-spin-flip reflectivities $R^{++}$and $R^{--}$at low $k_{z \text { (in) }}$, but which disappears at higher $k_{z}$. This is a signature of the Zeeman effect, which will be most pronounced when the Zeeman energy is comparable to the kinetic energy along the scattering direction.

The best indication that this splitting is a result of the Zeeman effect is to compare with data fitted to a model with no Zeeman energy included; these are the curves shown in Fig. 3.

In Figs. 4 and 3 there is an apparent horizontal shift between the two spin-flip reflectivities. This is entirely due to the choice of plotting these data as a function of $2 k_{z \text { (in) }}$. If we had chosen to plot the data versus the total momentum transfer $Q$, the features would be mostly aligned, but the advantage of plotting the data the way we have is that the scattering sum rules are more apparent; for an incident beam $I^{-}$at low angles where the scattering is strong, we can clearly see the non-spinflip reflectivity $R^{--}$has a dip when $R^{-+}$has a peak (a similar correspondence is seen between $R^{++}$and $R^{+-}$).

\section{Conclusions}

We have described a procedure for measuring polarized neutron reflectivity in high fields, including important changes to the modeling and instrument configuration due to Zeeman shifts in the energy and angle of spin-flip scattered neutrons. These considerations will be important for the characterization of thin films with a large magnetic anisotropy, which are a component of a growing number of technologically relevant systems.

A data-modeling package with the necessary modifications for this type of measurement has been demonstrated to provide accurate quantitative fits of a test system, and this software is now readily available to the research community (Kienzle, 2015). The deviations from non-Zeeman-corrected polarized specular neutron modeling are most pronounced where the spin-flip scattering is most intense.

\section{Acknowledgements}

YL is supported by the Division of Scientific User Facilities of the Office of Basic Energy Sciences, US Department of Energy.

\section{References}

Dura, J., Pierce, D., Majkrzak, C., Maliszewskyj, N., McGillivray, D., Lösche, M., O’Donovan, K. V., Mihailescu, M., Perez-Salas, U., Worcester, D. \& White, S. H. (2006). Rev. Sci. Instrum. 77, 074301.

Felcher, G., Adenwalla, S., De Haan, V. \& Van Well, A. (1995). Nature, 377, 409-410.

Felcher, G., Adenwalla, S., De Haan, V. \& Van Well, A. (1996). Phys. B Condens. Matter, 221, 494-499.

Fitzsimmons, M. R., Park, S., Dumesnil, K., Dufour, C., Pynn, R., Borchers, J. A., Rhyne, J. J. \& Mangin, Ph. (2006). Phys. Rev. B, 73, 134413.

Kienzle, P. (2015). Refl1D Package for Fitting Reflectivity Data, http:// ncnr.nist.gov/reflpak.

Kirby, B., Kienzle, P., Maranville, B., Berk, N., Krycka, J., Heinrich, F. \& Majkrzak, C. (2012). Curr. Opin. Colloid Interface Sci. 17, 44-53.

Kozhevnikov, S., Ott, F. \& Radu, F. (2012). J. Appl. Cryst. 45, 814-825.

Kruijs, R. W. E. van de, Fredrikze, H., Rekveldt, M., van Well, A., Nikitenko, Y. \& Syromyatnikov, V. (2000). Phys. B Condens. Matter, 283, 189-193.

Liu, Y., te Velthuis, S. G. E., Jiang, J. S., Choi, Y., Bader, S. D., Parizzi, A. A., Ambaye, H. \& Lauter, V. (2011). Phys. Rev. B, 83, 174418.

Majkrzak, C., O'Donovan, K. \& Berk, N. (2006). Neutron Scattering from Magnetic Materials, edited by T. Chatterji, pp. 397-471. Amsterdam: Elsevier Science. 